\title{
Structural dynamic modification
}

\author{
A SESTIERI \\ Dipartimento di Meccanica e Aeronautica, Università "La Sapienza”, Rome, \\ Italy \\ e-mail: aldo@sestieri.ing.uniromal.it
}

\begin{abstract}
Vibration and acoustic requirements are becoming increasingly important in the design of mechanical structures, but they are not usually of primary concern in the design process. So the need to vary the structural behaviour to solve noise and vibration problems often occurs at the prototype stage, giving rise to the so-called structural modification problem. In this paper, the direct problem of determining the new response of a system, after some modifications are introduced into the system, is analysed using two different databases: the modal database and the frequency response function database. The limitations of the modal database are discussed. Structural modifications that can be accounted for are lumped masses, springs, dampers and dynamic absorbers.
\end{abstract}

Keywords. Structural dynamic modification; structural design; dynamic design; SDM using FRF.

\section{Introduction}

The problem of varying the dynamic behaviour of structures to reduce the vibration response or control the noise radiated by vibrating surfaces is an exciting challenge. This is particularly true if we refer to systems that were not originally designed to fulfil these requirements. In fact, vibration and acoustics are not usually the primary concern of a design project, although they increasingly become the focus of the problem once the system has been built up. Unfortunately, the control of noise and vibration at this last stage, often at project completion, is a very complex problem, either because the practical actions are limited or because significant changes to the main features of the system are not permitted. Moreover, when the considered situation is a running system, such as an engine, a plant etc., the knowledge of the main dynamic parameters is poor and the system must be supposed to be a black box, whose dynamics may be characterized by (experimental) modal testing.

Structural modification (SM) is a procedure aimed at identifying the changes required in a structural system to modify its dynamic behaviour (natural frequencies, structural modes,

Part of this paper is drawn from an earlier paper, A Sestieri, W D'Ambrogio 1989 A modification method for vibration control of structures. Mech. Syst. Signal Process. 3: 229-253, permission for using which was obtained from Academic Press by the author. 
frequency response) in the desired direction. Enhancement of the structural or acoustic response is one of the common goals of structural modification processes and can be related to any of the following elements: the source, the transmission path or the noise radiating component.

Two different problems are usually considered - the direct problem and the inverse problem. The direct problem consists in determining the effect of already established modifications. This is a verification problem aimed at establishing the efficiency of given changes on the dynamic behaviour of the considered system. The inverse problem, more complex, tries to identify, in the framework of a given set of possible modifications, the most appropriate changes required to obtain the desired dynamic behaviour. Therefore this is a typical design problem, consisting in specifying the required tools to obtain an improvement of the structural or acoustic response.

Data describing the original vibrating structure, whose dynamic behaviour is modified, can be obtained either from an experimental database or from theoretical analysis using a finite element model of the system.

If a finite element database is available, the SM problem does not require an excessive effort. But neither a finite element database nor the modal parameters of the system are generally known, especially if a complex system is considered. This makes the problem difficult to tackle. Therefore in recent years a great deal of attention has been devoted to black box structures, dynamically identified through modal testing. The object of this rather widespread effort was mainly the identification of spatial (elastic characteristics - i.e. mass and stiffness matrices) and/or modal parameters, in order to acquire some knowledge of the structure upon which to perform efficient modification. Since identification of spatial parameters derived from an incomplete "mobility" model is a highly ill-conditioned problem, most of the work has been concentrated on modal parameters. Excluding particular situations (high modal density, strongly coupled modes or high non-proportional damping), modal parameters can be identified quite efficiently, at least in the frequency band tested, and will produce useful albeit incomplete modal models. Once such a model is available, many interesting structural modification algorithms can be developed and used with some advantage.

Due probably to the degree of development of modal knowledge, most of the modification formulations apply to changes of modal characteristics, i.e. resonance shifts, $Q$-factor reduction, optimal placement of nodal points etc., and the consequent synthesis is, in fact, usually called "modal synthesis". This is not necessarily a limit although it does not complete the wide field of applications that can be expected from structural modification. Among these, some concern the need for reducing the vibration behaviour of particular points of the structure in the frequency band of interest, or the possibility of narrowing the rigid body natural frequencies of a suspended system to achieve the maximum isolation efficiency (Johnson \& Subhedar 1979).

Moreover, the incomplete modal model, identified from a continuous structure, severely affects the results of structural modification. Wei et al (1987) showed that when using modal parameters, the accuracy of the modified dynamic properties is largely affected by the quality of the modal model derived from experimental data. Elliot \& Mitchell (1987), and Braun \& Ram (1987), discussed the effect of modal truncation on structural and modal modification. The former authors showed that the major role on the effectiveness of hardware modifications is played by a selected number of modes rather than by the quantity of modes included in a database. Braun \& Ram (1987) focused on the impossibility of obtaining an exact solution for structural modification when using an incomplete set of 
eigenvectors. O'Callahan et al (1987) emphasized the effect of rigid body modes on structural variations, and Wang et al (1987) discussed the effect of close modes and incorrrect prediction of damping on sensitivity analysis.

For the above reasons, another modification approach is presented here (D'Ambrogio \& Sestieri 1987; Sestieri \& D' Ambrogio 1988, 1989). The method avoids a modal identification process and the pitfalls deriving from it. Moreover it may be used to handle useful applications and permits optimal modifications to be accomplished with different dynamic requirements.

The modifications considered are lumped modifications, which consist of any combination of lumped masses, stiffnesses (obtained by springs connecting two different points of the structure or one point of the structure to an external fixed point) and damping.

\section{Direct problem}

The data necessary to solve the direct problem are dynamic behaviour of the original structure and knowledge of the modifications.

The dynamic behaviour of the structure can be expressed by a modal database, made of natural frequencies and modeshapes (real or complex) identified by a curve-fitting of experimental data or determined from a theoretical analysis, or by a frequency response function (FRF) database, determined experimentally or theoretically.

Here, the traditional approach will first be considered - the modal database being used to represent the dynamic behaviour of the system. Next, the approach based on the FRF database will be presented.

\subsection{Modal database}

The method is known in the technical literature as modal synthesis. Two cases can be considered depending on whether the modeshapes are real or complex. The case of real modes, related to an undamped or proportionally damped system, is analysed. The case of complex modes can be similarly discussed.

2.1a Real modes: The equation of motion of the original system is

$$
\mathbf{M} \ddot{\mathbf{x}}+\mathbf{C} \dot{\mathbf{x}}+\mathbf{K x}=\mathbf{f} \quad \text { with } \quad \mathbf{C}=\alpha \mathbf{M}+\beta \mathbf{K} .
$$

The undamped homogeneous equation

$$
\mathbf{M} \ddot{\mathbf{x}}+\mathbf{K x}=0,
$$

provides the eigenvalue problem

$$
(\mathbf{K}-\lambda \mathbf{M}) \phi=0 .
$$

Its solution yields the eigenvalues $\Lambda$ and the eigenvectors (modes) $\Phi$,

$$
\boldsymbol{\Lambda}=\left[\begin{array}{ccc}
\omega_{1}^{2} & & \\
& \ddots & \\
& & \omega_{N}^{2}
\end{array}\right], \quad \boldsymbol{\Phi}=\left[\phi_{1} \phi_{2} \cdots \phi_{N}\right] .
$$


The eigenvectors satisfy the orthonormal conditions

$$
\boldsymbol{\Phi}^{T} \mathbf{M} \boldsymbol{\Phi}=\mathbf{I}, \quad \boldsymbol{\Phi}^{T} \mathbf{K} \boldsymbol{\Phi}=\boldsymbol{\Lambda}, \quad \boldsymbol{\Phi}^{T} \mathbf{C} \boldsymbol{\Phi}=\alpha \mathbf{I}+\beta \boldsymbol{\Lambda}=\mathbf{\Xi} .
$$

Using the coordinate transformation $\mathbf{x}=\mathbf{\Phi q}$ in the equation of motion, and premultiplying by $\boldsymbol{\Phi}^{T}$ one obtains:

$$
\boldsymbol{\Phi}^{T} \mathbf{M \Phi} \ddot{\mathbf{q}}+\boldsymbol{\Phi}^{T} \mathbf{C} \Phi \dot{\mathbf{q}}+\boldsymbol{\Phi}^{T} \mathbf{K} \boldsymbol{\Phi} \mathbf{q}=\boldsymbol{\Phi}^{T} \mathbf{f} .
$$

On using the orthonormal conditions, the previous equation becomes,

$$
\ddot{\mathbf{q}}+\Xi \dot{\mathbf{q}}+\Lambda \mathbf{q}=\boldsymbol{\Phi}^{T} \mathbf{f}
$$

As is known, this is an uncoupled system of equations.

By indicating by $\mathbf{\Delta M}, \Delta \mathbf{K}$ and $\Delta \mathbf{C}(=\alpha \mathbf{\Delta M}+\beta \Delta \mathbf{K})$ the matrices encompassing the modifications of mass, stiffness and damping, whose elements are computed in $\S 2$, the equation of motion of the modified structure can be written as,

$$
(\mathbf{M}+\Delta \mathbf{M}) \ddot{\mathbf{x}}+(\mathbf{C}+\Delta \mathbf{C}) \dot{\mathbf{x}}+(\mathbf{K}+\Delta \mathbf{K}) \mathbf{x}=\mathbf{f} .
$$

Using again the coordinate transformation $\mathbf{x}=\boldsymbol{\Phi q}$ and premultiplying by $\boldsymbol{\Phi}^{T}$ one has,

$$
\left(\mathbf{I}+\boldsymbol{\Phi}^{T} \Delta \mathbf{M \Phi}\right) \ddot{\mathbf{q}}+\left(\boldsymbol{\Xi}+\boldsymbol{\Phi}^{T} \Delta \mathbf{C} \boldsymbol{\Phi}\right) \dot{\mathbf{q}}+\left(\boldsymbol{\Lambda}+\boldsymbol{\Phi}^{T} \Delta \mathbf{K} \boldsymbol{\Phi}\right) \mathbf{q}=\boldsymbol{\Phi}^{T} \mathbf{f} .
$$

It is worthwhile pointing out that the matrices,

$$
\Delta \tilde{\mathbf{M}}=\Phi^{T} \Delta \mathbf{M \Phi} \quad \Delta \tilde{\mathbf{C}}=\Phi^{T} \Delta \mathbf{C} \Phi \quad \Delta \tilde{\mathbf{K}}=\Phi^{T} \Delta \mathbf{K} \Phi,
$$

are not usually diagonalised by the eigenvectors of the original structure. The equations of motion written in the modal coordinates of the original structure are then coupled:

$$
(\mathbf{I}+\Delta \tilde{\mathbf{M}}) \ddot{\mathbf{q}}+(\boldsymbol{\Xi}+\Delta \tilde{\mathbf{C}}) \dot{\mathbf{q}}+(\boldsymbol{\Lambda}+\Delta \tilde{\mathbf{K}}) \mathbf{q}=\boldsymbol{\Phi}^{T} \mathbf{f} .
$$

Therefore it is necessary to study the undamped homogeneous problem,

$$
(\mathbf{I}+\Delta \tilde{\mathbf{M}}) \ddot{\mathbf{q}}+(\boldsymbol{\Lambda}+\Delta \tilde{\mathbf{K}}) \mathbf{q}=0
$$

and the related eigenvalue problem,

$$
\left[(\boldsymbol{\Lambda}+\boldsymbol{\Delta} \tilde{\mathbf{K}})-\lambda^{I}(\mathbf{I}+\boldsymbol{\Delta} \tilde{\mathbf{M}})\right] \phi^{I}=0 .
$$

It provides the new eigenvalues $\Lambda^{I}$ and the new eigenvectors $\boldsymbol{\Phi}^{I}$, that uncouple the equations of motion by means of the new transformation $\mathbf{q}=\boldsymbol{\Phi}^{I} \mathbf{q}^{I}$. In fact, the following orthonormal conditions hold,

$$
\left(\boldsymbol{\Phi}^{I}\right)^{T}(\mathbf{I}+\boldsymbol{\Delta} \tilde{\mathbf{M}}) \boldsymbol{\Phi}^{I}=\mathbf{I}, \quad\left(\boldsymbol{\Phi}^{I}\right)^{T}(\boldsymbol{\Xi}+\boldsymbol{\Delta} \tilde{\mathbf{C}}) \boldsymbol{\Phi}^{I}=\boldsymbol{\Xi}^{I}, \quad\left(\boldsymbol{\Phi}^{I}\right)^{T}(\boldsymbol{\Lambda}+\Delta \tilde{\mathbf{K}}) \boldsymbol{\Phi}^{I}=\boldsymbol{\Lambda}^{I},
$$

and the uncoupled set of equations is,

$$
\ddot{\mathbf{q}}^{T}+\boldsymbol{\Xi}^{I} \dot{\mathbf{q}}^{I}+\Lambda^{I} \mathbf{q}^{I}=\boldsymbol{\Phi}^{I^{T}} \boldsymbol{\Phi}^{T} \mathbf{f} .
$$

The natural frequencies of the modified structure can be obtained from the eigenvalue matrix $\Lambda^{I}$, whilst the modeshapes of the modified structure are given by, $\boldsymbol{\Psi}=\boldsymbol{\Phi} \boldsymbol{\Phi}^{I}$. 
In fact, the transformation $\mathbf{x}=\boldsymbol{\Psi} \mathbf{q}^{I}$ from the physical coordinates to the modal coordinates of the modified structure can be written as, $\mathbf{x}=\boldsymbol{\Phi q}=\boldsymbol{\Phi} \boldsymbol{\Phi}^{I} \mathbf{q}^{I}$, and the matrix of the new modeshapes can be put in the form, $\boldsymbol{\Psi}=\left[\begin{array}{lll}\psi_{1} & \psi_{2} & \cdots\end{array} \psi_{N}\right]$.

Note that the new modes are a linear combination of the modes of the original structure. In fact the general element $\psi_{r s}$ of the matrix $\boldsymbol{\Psi}$ is, $\psi_{r s}=\sum_{l=1}^{N} \phi_{r l} \phi_{l s}^{I}$ equivalent to: $\psi_{s}=\sum_{l=1}^{N} \phi_{l s}^{I} \phi_{l}$.

It should be noted that the computation of the modified natural frequencies and modeshapes through (12) does not require the knowledge of the mass, stiffness and damping matrices $\mathbf{M}, \mathbf{K}$ and $\mathbf{C}$ of the original structure. Therefore it is possible to determine the modal parameters of the modified structure provided that the natural frequencies and vibrational modes of the original structure, as well as the matrices $\Delta \mathbf{M}$ and $\Delta \mathbf{K}$, are known. The modal parameters can be determined experimentally, i.e. identified from experimental FRFs.

The problem (12) may be a problem of lower order than the original one. The order of the problem is equal to the used number of modes of the original system. If $m=N$, the new modal parameters are determined exactly. On the contrary, if $m<N$, the new modal parameters are only approximate due to the truncation effect. This is the most common situation. In fact, it is not possible to identify the whole set of modal parameters of a continuous system, but only those included in a given frequency bandwidth. In other words,

(a)

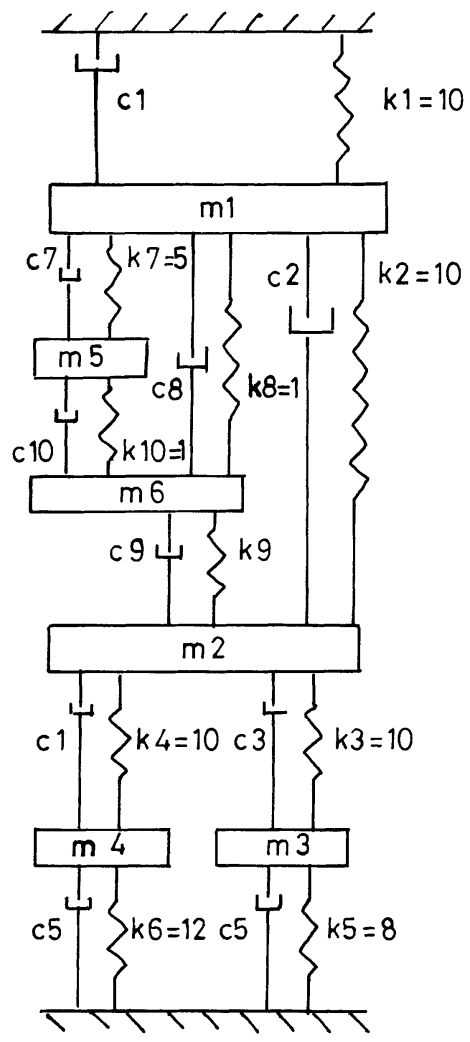

(b)

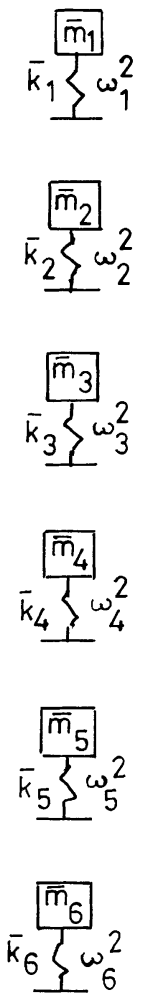

Figure 1. Six DOFs system. (a) Physical, and (b) modal models. 
for continuous systems, $N$ is infinite. Even in the so-called reanalysis problem in which a finite element model is used with $N$ having a finite value, the use of relationship (12) is useful only if, from a computational point of view, the computation is less time-consuming than the analysis of a new finite element model. This happens when in the reanalysis problem a number of modes $m<N$ is used.

It was observed that the mode of truncation is a source of error in the evaluation of the modal parameters of the modified structure. This effect will now be shown with reference to a particular case.

In figure 1, a six-degrees-of-freedom (6 DOF) lumped system is considered. In the same figure the modal model of such system is shown, representing the uncoupled system (6).

In figure $2 \mathrm{a}$, the system of figure 1 has been modified by adding a spring of stiffness $K=10 \mathrm{~N} / \mathrm{m}$ between the masses 1 and 3. The matrix $\Delta \mathbf{K}$ is given by (see $\S 3$ ):

$$
\Delta \mathbf{K}=\left[\begin{array}{cccccc}
10 & 0 & -10 & 0 & 0 & 0 \\
0 & 0 & 0 & 0 & 0 & 0 \\
-10 & 0 & 10 & 0 & 0 & 0 \\
0 & 0 & 0 & 0 & 0 & 0 \\
0 & 0 & 0 & 0 & 0 & 0 \\
0 & 0 & 0 & 0 & 0 & 0
\end{array}\right]
$$

(a)

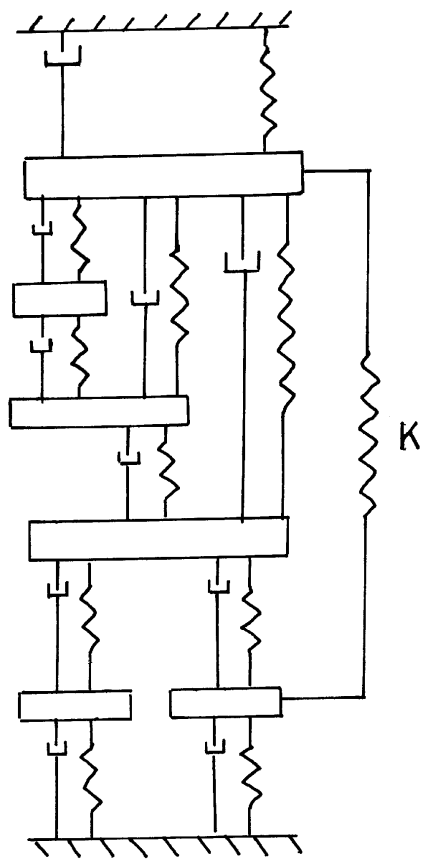

(b)

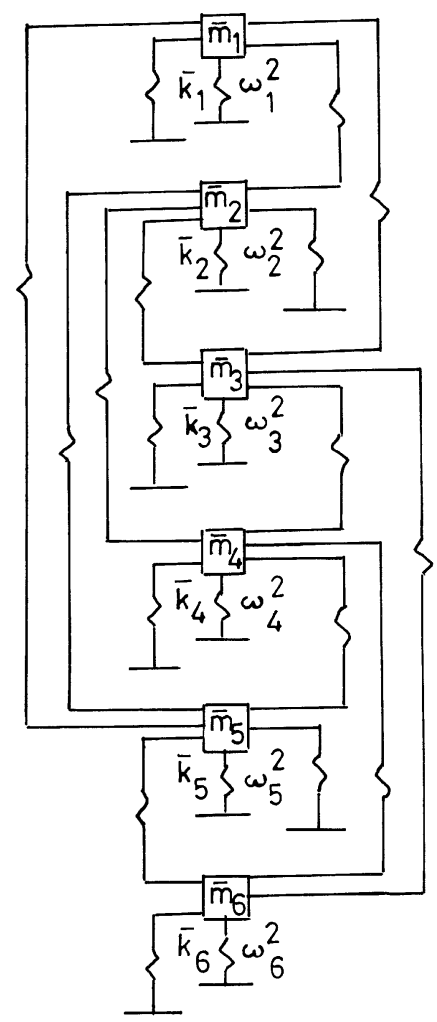

Figure 2. (a) Modified 6 DOFs system, (b) modal model of the modified system 
(a)

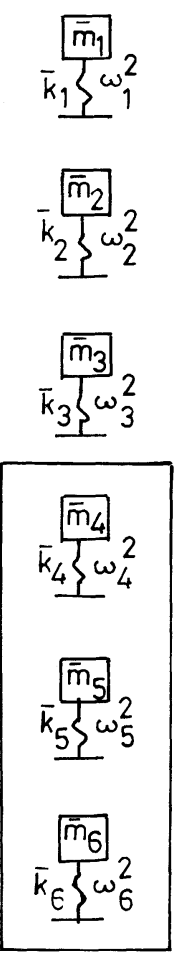

(b)

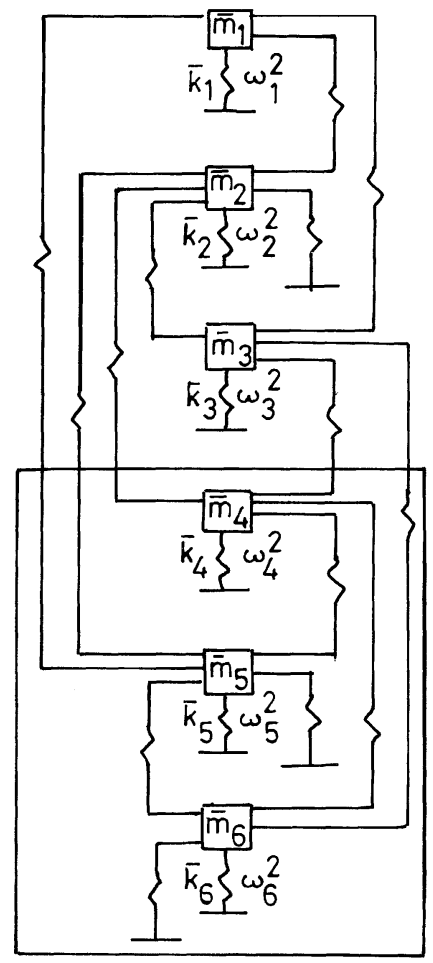

Figure 3. Truncation effect. (a) Unmodified and (b) modified modal models.

The homogeneous problem of the modified system is then,

$$
\ddot{\mathbf{q}}+(\boldsymbol{\Lambda}+\Delta \tilde{\mathbf{K}}) \mathbf{q}=0 .
$$

In figure $2 b$, the modal model of the modified system, represented by (16), is shown. The static coupling is provided by the springs interconnecting the modal masses.

The truncation effect is shown in figure 3. If the fourth, fifth and sixth modes of the original system are neglected, the correspondent modal model is made of the first three modes only. On the contrary, in the modal model of the modified system the truncation of the fourth, fifth and sixth modes does not permit us to account for the coupling terms among the first three modes and the omitted ones. These terms, appearing in the intersection between the first three rows and the last three columns of the matrix $\Delta \mathbf{K}$, are not at all negligible with respect to those of the last three columns.

It can be observed that the truncation effect cannot be easily quantified a priori, as it was emphasized by several researchers, although some of them tried to provide some kind of estimate on the maximum error introduced by the truncation.

\subsection{Frequency response function database}

The difficulty of identifying the modal parameters, especially for complex structures and/or high modal densities, and the arguments presented on the errors arising in the use of 
the modal model for the structural modification problem, suggest the avoidance, whenever possible, of the use of such a model.

Then it may be appropriate to use directly the frequency response function (FRF) matrix of the structure $\mathbf{H}_{0}(\omega)$, experimentally determined for $N$ degrees of freedom, and having the dimensions $N \times N$. From the modal parameters, any element of the FRF matrix can be reconstructed provided that the $N$ elements of a row or column of such matrix have been experimentally determined. However, in this case, to avoid the problems related to the identification and use of modes, it is necessary to measure the whole elements $N^{2}$ of the FRF matrix. Nevertheless, if the structure is linear, the matrix $\mathbf{H}_{0}(\omega)$ is symmetric due to the reciprocity theorem. Consequently the elements that must be actually determined are only $N \times(N+1) / 2$ (elements of the upper or lower triangular of the matrix), instead of $N^{2}$.

It is now shown how the FRF matrix of the modified structure can be determined from the knowledge of the original structure and modifications.

Using the FRF matrix, the response of the original structure, described by $\mathbf{u}_{0}(\omega)$, to an external excitation, described by $\mathbf{f}(\omega)$, is given by:

$$
\mathbf{u}_{0}(\omega)=\mathbf{H}_{0}(\omega) \mathbf{f}(\omega) .
$$

In (17) $\mathbf{H}_{0}(\omega)$ is the FRF matrix of the original structure, whose elements can be receptances, mobilities or inertances. Correspondingly, the elements of $\mathbf{u}_{0}(\omega)$ are displacements, velocities or accelerations respectively, of the $N$ DOFs considered, while the elements of $\mathbf{f}(\omega)$ are the forces acting on the structure at those DOFs.

Using, at least formally, the impedance-type matrix of the structure $\mathbf{B}_{0}(\omega)=\mathbf{H}_{0}^{-1}(\omega)$, the equation of motion of the original structure in the frequency domain is:

$$
\mathbf{f}(\omega)=\mathbf{B}_{0}(\omega) \mathbf{u}_{0}(\omega) .
$$

Let the modifications be described by the matrix $\Delta \mathbf{B}(\omega)$, that can be written, for the dynamic stiffness:

$$
\Delta \mathbf{B}(\omega)=\Delta \mathbf{K}+j \omega \mathbf{\Delta} \mathbf{C}-\omega^{2} \boldsymbol{\Delta} \mathbf{M} .
$$

If the modifications involve DOFs in which the $\mathbf{H}_{0}(\omega)$ matrix has been measured, then the equation of motion of the modified structure, excited by the same forces $\mathbf{f}(\omega)$, is written as:

$$
\mathbf{f}(\omega)=\left[\mathbf{B}_{0}(\omega)+\Delta \mathbf{B}(\omega)\right] \mathbf{u}(\omega),
$$

in which $\mathbf{u}(\omega)$ is the response of the modified structure. Since the FRF matrix of the modified structure is still unknown, the response $\mathbf{u}(\omega)$ is:

$$
\mathbf{u}(\omega)=\mathbf{H}(\omega) \mathbf{f}(\omega),
$$

in which it is:

$$
\mathbf{H}(\omega)=\left[\mathbf{B}_{0}(\omega)+\Delta \mathbf{B}(\omega)\right]^{-1} .
$$

Keeping in mind that the impedance-type matrix of the original structure is not practically available, one can write:

$$
\mathbf{H}(\omega)=\left[\mathbf{H}_{0}^{-1}(\omega)+\boldsymbol{\Delta} \mathbf{B}(\omega)\right]^{-1} .
$$


It is worthwhile pointing out that it is convenient not to use (23) directly, because two matrix inversions are required to compute $\mathbf{H}(\omega)$. It is more convenient to premultiply (20) by $\mathbf{H}_{0}(\omega)$, thus obtaining:

$$
\mathbf{H}_{0}(\omega) \mathbf{f}(\omega)=\mathbf{H}_{0}(\omega)\left[\mathbf{B}_{0}(\omega)+\Delta \mathbf{B}(\omega)\right] \mathbf{u}(\omega)=\left[\mathbf{I}+\mathbf{H}_{0}(\omega) \boldsymbol{\Delta} \mathbf{B}(\omega)\right] \mathbf{u}(\omega),
$$

in which $\mathbf{I}$ is the identity matrix. From (24) one obtains:

$$
\mathbf{u}(\omega)=\left[\mathbf{I}+\mathbf{H}_{0}(\omega) \Delta \mathbf{B}(\omega)\right]^{-1} \mathbf{H}_{0}(\omega) \mathbf{f}(\omega) .
$$

By comparing (25) and (21) one obtains:

$$
\mathbf{H}(\omega)=\left[\mathbf{I}+\mathbf{H}_{0}(\omega) \Delta \mathbf{B}(\omega)\right]^{-1} \mathbf{H}_{0}(\omega) .
$$

Equation (26), providing the FRF matrix of the modified structure in function of the original FRF $\mathbf{H}_{0}(\omega)$ and the impedance-type matrix of the modifications $\Delta \mathbf{B}(\mathbf{x}, \omega)$, is the prediction relationship we are looking for. With respect to (23), it involves a unique matrix inversion, so that it is computationally more efficient. Moreover it is unlikely that the matrix to invert in (26) is ill-conditioned, as it is the sum of two matrices. The first is the identity matrix, the second is the product of the original FRF matrix by the modification matrix. Provided that the proposed modifications are small with respect to the original physical parameters of the structure, the elements of the product matrix $\mathbf{H}_{0}(\omega) \Delta \mathbf{B}(\mathbf{x}, \omega)$ would be small with respect to those of the unit matrix. Then the optimal behaviour of the $\mathbf{I}$ matrix with respect to the inversion operation is lightly affected by the second term.

It is worth pointing out that, for the given set of modifications, the computation performed in (26) must be repeated for all the frequencies of interest. They are established by the analyst among those used to determine $\mathbf{H}_{0}(\omega)$ experimentally. Because of the digital computers normally used in obtaining experimental data, these frequencies represent a discrete set of values.

The computational burden of this operation is not low. In fact the algorithms performing the inversion of a matrix of order $N \times N$ involve a number of operations proportional to $N^{3}$. Therefore, reduction in the order of the matrix to be inverted provides a reduction in the computational time that increases with the initial value of $N$. For example, passing from $N$ to $N-1$ one has a reduction in the number of operations of order $N^{2}$. In fact

$$
N^{3}-(N-1)^{3}=3 N^{2}-3 N+1 .
$$

Significant simplification and consequent reduction of time computation can be then obtained if there are only $b$ DOFs involved in the modifications $(b<N)$. In such a case the matrix $\Delta \mathbf{B}(\omega)$ can be written as

$$
\Delta \mathbf{B}_{N \times N}=\left[\begin{array}{cc}
0_{a a} & 0_{a b} \\
0_{b a} & \boldsymbol{\Delta} \mathbf{B}_{b b}
\end{array}\right] .
$$

It can be shown that in this case any element of the modified FRF matrix $\mathbf{H}(\omega)$ can be obtained by inverting a matrix of order $b$ instead of $N$. The result is, after some mathematics (D’Ambrogio 1990),

$$
\left[\begin{array}{ll}
\mathbf{H}_{a a} & \mathbf{H}_{a b} \\
\mathbf{H}_{b a} & \mathbf{H}_{b b}
\end{array}\right]=\left[\begin{array}{ll}
\mathbf{H}_{0 a a} & \mathbf{H}_{0 a b} \\
\mathbf{H}_{0 b a} & \mathbf{H}_{0 b b}
\end{array}\right]-\left[\begin{array}{l}
\mathbf{H}_{0 a b} \\
\mathbf{H}_{0 b b}
\end{array}\right] \Delta \mathbf{B}_{b b}\left[\mathbf{I}_{b b}+\mathbf{H}_{0 b b} \Delta \mathbf{B}_{b b}\right]^{-1}\left[\mathbf{H}_{0 b a} \mathbf{H}_{0 b b}\right] .
$$


Equation (29) permits us to obtain any element of the modified FRF matrix by inverting a matrix of order $b \times b$ instead of $N \times N$, with significant advantage from a computational point of view.

\section{Description of modifications}

Especially in the inverse problem, it is important that the proposed modifications be physically built up from their description through a given number of variables. In fact their values are determined to provide the required dynamic behaviour of the modified structure. Therefore it is necessary that, according to the solution provided by the inverse problem, such modifications be effectively obtained.

To this end, it is convenient that the structural modifications be described by the spatial (or physical) model, that is by an impedance-type matrix $\Delta \mathbf{B}(\mathbf{x}, \omega)$, in which the vector $\mathbf{x}$ collects the variables characterizing the set of involved modifications.

Distributed modifications - such as beams, plates, stiffners - although practically very useful, cannot be easily considered by the proposed procedure. This difficulty arises because the forces exchanged between the structure and the modifications, that are important to evaluate the impedance-type matrix $\Delta \mathbf{B}(\mathbf{x}, \omega)$, also involve the rotational degrees of freedom. Rotations are not usually considered in the experimentally determined FRFs because of the lack of suitable transducers capable of measuring these quantities efficiently. Consequently, since a one-to-one correspondence between elements of $\Delta \mathbf{B}(\mathbf{x}, \omega)$ and $\mathbf{H}_{0}(\omega)$ exists, elements involving rotational DOFs cannot be present in $\Delta \mathbf{B}(\mathbf{x}, \omega)$. It is not then allowed, at this stage, to consider directly the effects of rotations and moments.

For this reason, and considering that very often the structural modification is requested at the final stage of the design process, thus not allowing significant alteration of the general architecture of the system, it is convenient here to consider only elementary modifications, represented by lumped models.

\subsection{Elementary modifications}

The considered elementary modifications are:

- concentrated masses;

- one-dimensional springs between two points of the structure or between the structure and an external fixed point;

- viscous dampers between two points of the structure or between the structure and an external fixed point;

- dynamic absorbers connected to a point of the structure;

- continuous rods of uniform cross-section between two points of the structure or between the structure and an external fixed point.

If $N_{m}, N_{k}, N_{c}, N_{d}$ and $N_{a}$ are the number of masses, springs, viscous dampers, dynamic absorbers and rods respectively, the total number of elementary modifications is simply given by,

$$
N_{M}=N_{m}+N_{k}+N_{c}+N_{d}+N_{a} .
$$

The variables necessary to represent any of the above elementary modifications are: a single variable for any concentrated mass (mass value $m$ ); a single variable for any 
one-dimensional spring (stiffness value of the spring $k$ ); a single variable for any viscous damper (damping value $c$ ); three variables for any dynamic absorber (mass value $m$, stiffness value $k$ and loss factor $\eta$ ); three variables for any rod (mass value $m$, static stiffness $k$ and loss factor $\eta$ ).

In order to work with non-dimensional quantities - a condition required in optimization problems to obtain numerical values of the same order - it is convenient to establish a reference value for any category of dimensional variable. Therefore, masses, stiffnesses and viscous dampings are suitably transformed into non-dimensional quantities through a reference mass $m_{0}$, a reference stiffness $k_{0}$ and a reference damping $c_{0}$, appropriately chosen by the analyst. Let $\mathbf{e}_{i}$ be the vector of dimension 1 or 3 , containing the non-dimensional variables, corresponding to the $i$ th element modification. The total number of variables that describe a given set of modifications is then given by:

$$
N_{V}=N_{m}+N_{k}+N_{c}+3\left(N_{d}+N_{a}\right) .
$$

The values of the non-dimensional variables corresponding to these modifications are contained in the vector $\mathbf{x}$, whose dimension is $N_{V}$ : for example, the first elements correspond to the masses, then the stiffnesses, the viscous dampings, the dynamic absorbers and, finally, to the rods.

If $\Delta \mathbf{B}\left(\mathbf{e}_{i}, \omega\right)$ is the matrix corresponding to the $i$ th element modification, the matrix $\Delta \mathbf{B}(\mathbf{x}, \omega)$, corresponding to the whole set of modifications, is simply given by the sum of the matrices of element modification, i.e.:

$$
\Delta \mathbf{B}(\mathbf{x}, \omega)=\sum_{i=1}^{N_{M}} \Delta \mathbf{B}\left(\mathbf{e}_{i}, \omega\right) .
$$

The elements of the impedance-type matrix $\Delta \mathbf{B}\left(\mathbf{e}_{i}, \omega\right)$, corresponding to the $i$ th considered element modification, can be easily determined as shown by Sestieri \& D'Ambrogio (1989).

The element modifications considered are:

- concentrated mass $m$, represented by the non-dimensional variable $\mathbf{e}_{i}=\hat{m}=m / m_{0}$;

- spring of stiffness $k$, represented by the non-dimensional variable $\mathbf{e}_{i}=\hat{k}=k / k_{0}$;

- viscous damping $c$, represented by the non-dimensional variable $\mathbf{e}_{i}=\hat{c}=c / c_{0}$;

- dynamic absorber of mass $m$, stiffness $k$ and loss factor $\eta$, represented by the nondimensional variables $\hat{m}=m / m_{0}, \hat{k}=k / k_{0}$ and $\eta$, so that $\mathbf{e}_{i}=\{\hat{m} \hat{k} \eta\}^{T}$;

- continuous rod of mass $m$, stiffness $k$ and loss factor $\eta$, represented by the non-dimensional variables $\hat{m}=m / m_{0}, \hat{k}=k / k_{0}$ and $\eta$, so that $\mathbf{e}_{i}=\{\hat{m} \hat{k} \eta\}^{T}$.

3.1a Concentrated mass: Adding or subtracting a concentrated mass affects the degree of freedom at the point where the mass is applied. In other words the mass exerts a force on the structure in the direction of the vibratory motion of such point, 'a priori' unknown. Therefore the matrix $\mathbf{\Delta} \mathbf{B}(\hat{m}, \omega)$ has a non-zero element on the diagonal, in correspondence of the DOF of the point where the mass is applied. It is shown by Sestieri \& D'Ambrogio (1989) that

$$
\Delta B_{r r}(\hat{m}, \omega)=-L_{r r}(\hat{m}, \omega)=-m \omega^{2} .
$$

It is then necessary to describe the effect of the mass $m$ by a single element of the matrix $\Delta \mathbf{B}$, because of the one-to-one correspondence with the matrix $\mathbf{H}_{0}$. 
$3.1 \mathrm{~b}$ One-dimensional spring: Adding an axial spring between two points of the structure or between a point of the structure and an external fixed point implies the application of a force along the axis of the spring, due to the motion of the points on the structure. Considering displacements along the $r$ and $s$ DOFs, the force $F_{a}^{B}$ acting on the structure along the spring axis is

$$
F_{a}^{B}(\hat{k}, \omega)=-k\left(u_{r} \cos \alpha_{r}-u_{s} \cos \alpha_{s}\right),
$$

where $\alpha_{r}$ and $\alpha_{s}$ are the angles between the spring's axis and the $r$ and $s$, respectively.

It is shown by Sestieri \& D'Ambrogio (1989) that, in this case,

$$
\begin{aligned}
& \Delta B_{r r}(\hat{k}, \omega)=k \cos ^{2} \alpha_{r}, \\
& \Delta B_{r s}(\hat{k}, \omega)=-k \cos \alpha_{r} \cos \alpha_{s}, \\
& \Delta B_{s r}(\hat{k}, \omega)=-k \cos \alpha_{r} \cos \alpha_{s}, \\
& \Delta B_{s s}(\hat{k}, \omega)=k \cos ^{2} \alpha_{s} .
\end{aligned}
$$

If the spring is connected to an external fixed point $u_{s}=0$, one easily determines:

$$
\Delta B_{r r}(\hat{k}, \omega)=k \cos ^{2} \alpha_{r} .
$$

Thus, in general, in the $\Delta \mathbf{B}(\hat{k}, \omega)$ matrix describing a spring between two points of the structure, there are four non-zero elements, whilst for a spring connected to an external point, only one is different from zero.

3.1c Viscous damping: The above developments, valid for the axial springs, can be repeated for a viscous damper. Therefore (Sestieri \& D'Ambrogio 1989) for a damper between two points of the structure,

$$
\begin{aligned}
& \Delta B_{r r}(\hat{c}, \omega)=j \omega c \cos ^{2} \alpha_{r}, \\
& \Delta B_{r s}(\hat{c}, \omega)=-j \omega c \cos \alpha_{r} \cos \alpha_{s}, \\
& \Delta B_{s r}(\hat{c}, \omega)=-j \omega c \cos \alpha_{r} \cos \alpha_{s}, \\
& \Delta B_{s s}(\hat{c}, \omega)=j \omega c \cos ^{2} \alpha_{s},
\end{aligned}
$$

and for a damper connected to an external point,

$$
\Delta B_{r r}(\hat{c}, \omega)=j \omega c \cos ^{2} \alpha_{i} .
$$

3.1d Dynamic absorber: A dynamic absorber affects a larger number of DOFs because at least, a new coordinate must be used to describe the motion of the mass of the absorber. Sestieri \& D'Ambrogio (1989) show that for the dynamic absorber, one has:

$$
\Delta B_{r r}\left(\{\hat{m} \hat{k} \eta\}^{T}, \omega\right)=-\frac{\omega^{2} m k(1+j \eta)}{k(1+j \eta)-\omega^{2} m} .
$$

3.1e Continuous rod: The difference between the scheme of an axial spring and the continuous rod is in the stiffness $k$ which is not sufficient to describe the dynamic behaviour of the rod. It is now necessary to introduce the inertial properties through its mass $m$ and, eventually, the dissipative properties through $\eta$. Sestieri \& D'Ambrogio (1989) 
show that for a rod connecting two points of the structure

$$
\begin{aligned}
& \Delta B_{r r}\left(\{\hat{m} \hat{k} \eta\}^{T}, \omega\right)=\left[k(1+j \eta)-m \omega^{2} / 3\right] \cos ^{2} \alpha_{r}, \\
& \Delta B_{r s}\left(\{\hat{m} \hat{k} \eta\}^{T}, \omega\right)=\left[-k(1+j \eta)-m \omega^{2} / 6\right] \cos \alpha_{r} \cos \alpha_{s}, \\
& \Delta B_{s r}\left(\{\hat{m} \hat{k} \eta\}^{T}, \omega\right)=\left[-k(1+j \eta)-m \omega^{2} / 6\right] \cos \alpha_{r} \cos \alpha_{s}, \\
& \Delta B_{s s}\left(\{\hat{m} \hat{k} \eta\}^{T}, \omega\right)=\left[k(1+j \eta)-m \omega^{2} / 3\right] \cos ^{2} \alpha_{s},
\end{aligned}
$$

and for a rod connected to an external fixed point,

$$
\Delta B_{r r}\left(\{\hat{m} \hat{k} \eta\}^{T}, \omega\right)=\left[k(1+j \eta)-m \omega^{2} / 3\right] \cos ^{2} \alpha_{r} .
$$

\section{Conclusions}

The problem of structural modifications was presented in the framework of two different approaches by using a modal database and an FRF database. The modal database presents more limitations than the FRF approach, due to truncation effects in the former case.

When dealing with structural modifications, either a direct problem or an optimization one can be considered. The first establishes the dynamic behaviour of the modified structure once a set of changes has been established. In this context both the modal and the FRF approaches are successful, although with the limitations examined for the modal database the inverse or optimization problem tries to find the optimum set of modifications that accomplishes the required dynamic behaviour of the system. The use of the FRF database is particularly appropriate to give a satisfactory solution of the problem, while this is not definitely the case with the modal approach.

\section{References}

Braun S, Ram Y M 1987 On structural modifications in truncated systems. Proc. 5th Int. Modal Anal. Conf. (London) 2: 1550-1556

D’Ambrogio W 1990 Some remarks about structural modifications involving additional degrees of freedom. Mach. Syst. Signal Process. 4: 95-99

D'Ambrogio W, Sestieri A 1987 Skipping model identification in performing structural modification. Proc. 12th Int. seminar on modal analysis, Leuven, S1-1

Elliot K B, Mitchell L D 1987 The effect of modal truncation on model modification. Proc. 5th Int. Modal Anal. Conf. (London) 1: 72-78

Johnson S R, Subhedar J W 1979 Computer optimization of engine mounting systems. SAE Paper No. 790974

O'Callahan J C, Chou C M, Avitabile P, Wu C H 1987 Consistent scaling of rigid body using experimental flexural modes. Proc. 5th Int. Modal Anal. Conf. (London) 2: 1538-1544

Sestieri A, D'Ambrogio W 1988 Why be model: i.e. how to avoid the use of modes in the modification of vibrating systems. Proc. 6th Int. Modal Anal. Conf. (Orlando) 2: 1100-1106

Sestieri A, D'Ambrogio W 1989 A modification method for vibration control of structures. Mech. Syst. Signal Process. 3: 229-254

Wang L, Heylen W, Sas P 1987 Accuracy of structural optimization techniques. Proc. 5th Int. Modal Anal. Conf. (London) 1: 65-71

Wei M L, Allemang R J, Brown D L 1987 Modal scaling considerations for structural modification application. Proc. 5th Int. Modal Anal. Conf. (London) 2: 1531-1537 